\title{
Lang anhaltende Schmerzen umfassend verstehen - was ist daran so schwierig?
}

Die Integration des Somatischen, Psychischen und Sozialen in das medizinische Konzept entspricht dem geistigen Band bei Goethe. Es fehlt häufig, wie Mephisto in Faust I dem Schüler erklärt: «Wer will was Lebendiges erkennen und beschreiben/Sucht erst den Geist heraus zu treiben/ Dann hat er die Teile in seiner Hand/Fehlt leider! nur das geistige Band». Dies führt zur Aufsplitterung in der Erfassung des Patienten. Der Arzt diagnostiziert und behandelt jetzt «Krankheiten» statt «Patienten mit Krankheiten».

Zur 14-tägigen Gruppensupervision bringt eine Hausärztin ihre 46-jährige Patientin mit. Sie weiss, dass ich für das Interview nur Name und Alter kennen möchte. So bleibe ich vorurteilsfrei und interviewe die Patientin, wie es Engel [1] sowie Adler und Hemmeler [2] vorgeschlagen haben.

\section{Das Interview}

Ich nehme nie Notizen während der Anamnese-Erhebung, damit ich mich empathisch in die Patientin versetzen kann. Auf meine Aufforderung, Platz zu nehmen, setzt sie sich mir schräg gegenüber, wendet den Kopf von mir weg der Gruppe zu. Sie seufzt und wirkt hilflos, seltsam lächelnd. Sie sei völlig erschöpft, müsse liegen; sie sei als Kind fünfmal an der Nase operiert worden. Sie leide an Asthma, Diskushernien, arbeite aber dennoch zu 20\% als Marktfrau. Dann nimmt sie ein A4-Blatt aus der Handtasche und liest zusätzliche Krankheiten vor: «Bauchkrämpfe, verschiedene Allergien, Neurodermitis, Fibromyalgie, Störung des Immunsystems, mehrere Selbstmordversuche. Gallenblasenentfernung.» Sie sei als Sturzgeburt zur Welt gekommen, ganz blau, man habe sie zur Seite gelegt, weil sie sterben werde. Die Eltern hätten sich scheiden lassen, als sie siebenjährig gewesen sei. Sie habe mehrere Vergewaltigungen erlebt. Ihr Ehemann habe sie häufig geschlagen. Sie habe trotzdem in der Ehe ausgehalten und habe zwei Söhne, das Mädchen dazwischen sei als Kind gestorben.

\section{Notizen nach Beendigung des 20-minütigen Gesprächs}

Ich dachte an W. Oslers «maladie du petit papier». Für ihn bedeutete das Papier Abwehr, indem es zwischen Patient und Arzt gestellt wird, sodass dieser sich aufs Papier und nicht auf das Erleben und die Geschichte konzentriert [3]). Ich teile diese Auffassung [4]. Es war mir unmöglich, die Symptome genau nach Lokalisation, Qualität, Intensität, verstärkenden und lindernden Faktoren, Begleitsymptomen, Beginn, zeitlichem Verlauf und sozialer Situation zu erfassen. Die Patientin verwirrte mich.

Überlegungen: Einige unbedeutende organische Veränderungen mögen vorliegen. Aber am wichtigsten sind die Verwirrung, die mich erfasst (Gegenübertragungsreaktion) und die unerfreuliche Kindheitsgeschichte. Die Patientin ist Opfer geworden, und für das weitere Leben «painprone», das heisst aus seelischen Gründen immer wieder oder dauernd zum Erleben von Schmerzen und anderen störenden Symptomen neigend [5], darunter zu Konversions-Symptomen (Ausdruck von psychischen Nöten in der Sprache des Körpers [6], Konditionierung auf Schmerz (z.B. wenn Zärtlichkeit nur erfahren wird, wenn das Kind krank oder verletzt ist) und Identifizierung mit einem kranken und geplagten Elternteil. Die «belle indifférence» ist dafür typisch. Die vage Beschreibung der Symptome passt auch dazu.

Nach der Supervision bat ich die Ärztin, mir die Akten zubringen. Ich erhielt 9,5 kg Unterlagen.

1986: mit 16 Jahren Unterleibsoperation an Ovar und Tube, Entzündung? (Es ist auffällig, wenn eine Frau schon im Teenageralter am Unterleib operiert wird). Sämtliche Bewegungen erfolgten verlangsamt, auffällig das Schmerz vermittelnde Gesicht und die Gestik.

1993: Arbeitgeber: mangelndes Arbeitsinteresse, häufige Absenzen, Kündigung. Postoperative Veränderungen palatal rechts mit Verdacht auf Verwachsungen des geschaffenen Ostiums.

1999: Thoraxschmerzen und Dyspnoe unklarer Genese, Herz-Doppler und Ergometrie normal.

2003: Verengte obere Atemwege, Asthma, Atembeschwerden, zahlreiche Allergien, (nicht nachgewiesen), Angstzustände, Konzentrationsstörungen, Rückenschmerzen. Auflistung von mehr als 30 Ärzten in der Vergangenheit.

2004: Im Rollstuhl, BSR 12, Ganzkörperszintigramm normal, keine Antikörper inkl. HLA B 27. Erstickungsängste, weitschweifig, inhaltlich unklar, mehrdeutig, diffuse Angaben, histrionisch, ängstlich, unsicher.

2008: Mikroadenom der Hypophyse, sekundäre Hypothyreose, anschliessend in zwei Zentralspitälern kein Adenom festgestellt, endokrine Werte normal. 2011: Gastroduodenoskopie: keine Erklärung der Symptome.

2012: Abdomensonografie normal.

2013: Bei IV-Abklärung mit eigenen Angaben: «Immunsystem-Problem, Osteoporose, Arthrose, Spondylarthrose, Fibromyalgie, Scheuermann, Arthritis, Skoliose, Beckenschiefstand, Schilddrüsenunterfunktion, bevorste- 
hender Diabetes, Diskushernien L 4/5/S1, Allergien, Asthma (Lungenfunktion normal), Neurodermitis, MagenDarm-Störungen. Schmerzfragebogen mit Anzeichnen der Lokalisationen: Kiefer, Augen und Augenhöhlen, Kopf, Schultern, Rücken, Kreuz, Ellenbogen, Handgelenk, Finger, Brust, Brustbein, Hüfte, Gesäss, Oberschenkel, Kniegelenk, Waden, Fussgelenke, Fusssolen. Symptomfragebogen: Müdigkeit, Schwindel, Vergesslichkeit, Zittern, Verstopfung, Verdauungsprobleme, Übersäuerung, Blähungen, vermehrtes Schwitzen, Herzklopfen und -rasen, Panik und Angstzustände, Reizbarkeit, Depressionen, trockener Mund und Lippen, Kloss und Schleim im Hals, trockene+ brennende Augenlieder, brennende Augen, Schlafstörungen Schwellungen, Taubheit, Stechen, Steifigkeit, Kribbeln, Zucken».

\section{Wie kann ein lang anhaltender Schmerz- zustand über Jahrzehnte undiagnostiziert bleiben und zu einer ungeheuren Anzahl von Abklärungen führen?}

Antworten: A) In der Ausbildung zum Arzt lernt der Student das biomedizinische Konzept kennen. Dieses beruht auf chemisch-physikalischer Evidenz. Der Patient wird, einem defekten Auto vergleichbar, zum offenen System, dem man die Motorhaube öffnen kann und nach Algorithmen defekte Teile sucht und repariert. Offen, weil jedermann ohne den Patienten zu kennen seine medizinische Arbeit verrichten kann. Dies ist in Notfällen wie bei einem Herzstilland gut. Die Wissenschaft vom Menschen ist die Wissenschaft von der Natur des Menschen, und diese ist eine biopsychosoziale, mit auch geschlossenen Teilen, nämlich dem Erleben und der Geschichte des Kranken, die nur mit einem geschulten Interview erfasst werden kann [7]. Die Integration des offenen und des geschlossenen Teils des Patienten muss im Erstinterview stattfinden, sonst fehlt das «geistige Band», indem der geschlossene Teil beiseitegelegt wird. Die Aufsplitterung in Teile erfolgt, der Patient wird von einem Spezialisten zum nächsten gewiesen, eine Synthese erfolgt nicht. William Osler sagte dazu: der gute Arzt behandelt Krankheiten, der sehr gute Arzt Patienten mit ihrer Krankheit. Selbstverständlich gibt es naturgemäss bessere und schlechtere Interviewer. Aber auch der Bessere bedarf der Förderung auf diesem Gebiet. Ein Vergleich mit dem Schach: Garry Kasparow war schon als Knirps ausser- ordentlich talentiert. Er begab sich dennoch zu Weltmeister Michail Botwinnik ins Training.

B) Daraus geht hervor, dass der Medizinstudent mehr Ausbildung im Interviewen, der Arzt-Patient-Beziehung und der psychischen Entwicklung des Kindes braucht, als die $5 \%$ an Stunden im Gesamtstudium [8].

Ist es nicht erfreulich, dass in einem Erstinterview ohne Vorkenntnisse über den Patienten in 20-30 Minuten sowohl wichtiges Biologisches und Psychosoziales integriert erfasst werden kann, wie bei unserer Patientin die Scheidung der Eltern, der sexuelle Missbrauch, die Brutalität des Ehepartners, die Suizidversuche, auf psychische Hintergründe hinweisende Mimik und Gestik? Das Erstgespräch, sachgerecht geführt, ist der Königsweg zum kranken Patienten. Es ermöglicht auch in anders gelagerten Fällen $85 \%$ der nötigen Daten zu erfahren, die Körperuntersuchung steuert durchschnittlich $10 \%$ bei, die Laboruntersuchungen $5 \%$. Bundesrat Bersets Verordnung, das Erstinterview sei auf 20 Minuten zu beschränken, verrät den Nichtkenner [9].

\section{Bibliografie}

1. Morgan WR, Engel GL: The clinical approach to the patient. Philadelphia; Saunders: 1969.

2. Adler RH, Hemmeler W: Anamnese und Körperuntersuchung, 3. Aufl. Stuttgart; Fischer: 1992.

3. Bean WB: Oslers aphorisms. New York; Schuman. 1950.

4. Adler RH:Patienten mit selbstverfassten Krankengeschichten: Symptom oder Zufall? Praxis 1986; 3: 41-44.

5. Engel GL: 'Psychogenic pain' and the pain-prone patient. Am J Med 1959; 26: 899-918.

6. Adler RH: Konversion. In: Uexküll Psychosomatische Medizin, 7. Aufl. München; Urban Fischer: 2010.691-699.

7. Adler RH, v Uexküll Th, Herrmann MJ: Die zwei Seiten medizinischer Evidenz. In: Herausforderung für die Biomedizin: Das biopsychosoziale Konzept. EMH 2017; 19-26.

8. Adler RH: 5 to 28 years follow-up of 99 residents trained in biopsychosozial internal medicine. PsychotherPsychosom 2008; 77: 126-127.

9. Adler RH. Bundesrat Bersets 20-Minuten Entscheid, oder: Der Königsweg zur Diagnose. SAEZ 2017; 889: 1539.

Prof. Dr. med. em. Rolf Adler

Leiserenweg 4

3122 Kehrsatz

michele.rolf.adler@gmail.com 